Publications of the Astronomical Society of the Pacific 108: 535-544, 1996 June

\title{
Parallelization and Algorithmic Enhancements of High-Resolution IRAS Image Construction
}

\author{
YU CAO AND THOMAS A. PRINCE \\ Division of Physics, Mathematics, and Astronomy, California Institute of Technology, Pasadena, California 91125 \\ Electronic mail: yucao@srl.caltech.edu, prince@srl.caltech.edu \\ Susan TEREBey AND CHARLES A. BEICHMAN \\ Infrared Processing and Analysis Center, California Institute of Technology, Pasadena, California 91125 \\ Electronic mail: st@ipac.caltech.edu, chas@ipac.caltech.edu \\ Received 1995 December 14; accepted 1996 March 22
}

\begin{abstract}
The Infrared Astronomical Satellite carried out a nearly complete survey of the infrared sky, and the survey data are important for the study of many astrophysical phenomena. However, many data sets at other wavelengths have higher resolutions than that of the coadded IRAS maps, and high-resolution IRAS images are strongly desired both for their own information content and their usefulness in correlation studies. The HIRES program was developed by the Infrared Processing and Analysis Center (IPAC) to produce high-resolution $\left(\sim 1^{\prime}\right)$ images from IRAS data using the maximum correlation method (MCM). We describe the port of HIRES to the Intel Paragon, a massively parallel supercomputer, other software developments for mass production of HIRES images, and the IRAS Galaxy Atlas, a project to map the Galactic plane at 60 and $100 \mu \mathrm{m}$. Images produced from the MCM algorithm sometimes suffer from visible striping and ringing artifacts. Correcting detector gain offsets and using a Burg entropy metric in the reconstruction scheme were found to be effective in suppressing these artifacts. A variation of the destriping algorithm was used to subtract zodiacal emission.
\end{abstract}

\section{INTRODUCTION}

The Infrared Astronomical Satellite (IRAS) provided our first comprehensive look at the infrared sky, producing a nearly complete survey at mid- to far-infrared wavelengths $(12,25,60$, and $100 \mu \mathrm{m})$ (Beichman 1987; Soifer et al. 1987; IRAS Catalogs and Atlases: Explanatory Supplement 1988). Images made from the IRAS survey data show a wealth of extended structure from star-forming regions and other components of the interstellar medium. A variety of studies exploiting the IRAS images have been made to date ranging from structure on a galactic scale to detailed studies of individual molecular clouds (e.g., Beichman et al. 1986; Weiland et al. 1986; Terebey and Fich 1986; Boulanger and Perault 1988; Sodroski et al. 1989; Scoville and Good 1989; Snell et al. 1989; Clemens et al. 1991; Wood et al. 1994). The strength of IRAS is the completeness of the survey. However, in many cases the spatial resolution of the comparison data sets at other wavelengths is better than for IRAS, and thus the $4^{\prime}-5^{\prime}$ resolution of the released IRAS images (the Infrared Sky Survey Atlas, Wheelock et al. 1994) can limit the comparison. The desire for higher spatial resolution combined with the paucity of new infrared satellite missions has inspired many efforts to extract high spatial-resolution information from the data (e.g., Bontekoe et al. 1994). The products most widely accessible to the US science community are the HIRES images distributed by the Infrared Processing and Analysis Center (IPAC), which are based on the maximum correlation method (MCM; Aumann et al. 1990). The HIRES images have been successfully used for a variety of galactic and extragalactic studies (Rice 1993; Surace et al. 1993; Terebey and Mazzarella 1994).

Application of the HIRES algorithm to the IRAS data has been limited largely by the computational resources available for HIRES processing. A $1^{\circ} \times 1^{\circ}$ field of typical scan coverage takes $1 \mathrm{hr}$ of CPU time on a Sun SPARCstation 10, for all four wavelength bands and 20 iterations (at which point artifacts limit further improvement of image quality). To overcome these CPU limitations we have undertaken the porting of the HIRES software to the Intel Delta and Paragon parallel supercomputers. HIRES processing is now feasible for large regions of the sky.

As part of a program in high-performance computational science and engineering, Caltech has developed significant software and hardware capabilities for massively parallel computing (also called concurrent supercomputing). Among the several concurrent computers currently available at Caltech is the 512-node Intel Touchstone Delta, a prototype parallel supercomputer with measured performance of 13 GFLOPS, 8 Gigabytes of memory, and 90 Gigabytes of disk. Upgraded resources include a 56-node and a 512-node Intel Paragon. The new 512-node Intel Paragon Model L38 has a peak speed of 38.4 GFLOPS, 16 Gigabytes of memory, and 14 RAIDs that control 67.2 Gigabytes of disk, one Ethernet node, two HIPPI nodes, and six service nodes. The high demand for HIRES images, along with the availability of parallel computing facilities, motivated the port of HIRES to the parallel supercomputers.

The development of new artifact reduction algorithms allows the iterative procedure to be carried much further, re- 
quiring more CPU time and further justifying the parallel computing approach.

These efforts made possible a large-scale mapping project: high-resolution IRAS mapping of the Galactic plane. The new IRAS Galaxy Atlas (IGA) maps will provide a 20 fold improvement in areal information content over current IRAS 60 and $100 \mu \mathrm{m}$ maps and will be valuable for a wide range of scientific studies, including: (1) the structure and dynamics of the interstellar medium (ISM); (2) cloud core surveys within giant molecular clouds; (3) determination of initial mass functions (IMFs) of massive stars; (4) study of supernova remnants (SNRs).

The IGA images will be made available on-line at IPAC. Additional information will come from combining the 60 and $100 \mu \mathrm{m}$ HIRES data with the images and catalogs being produced from the 12 and $25 \mu \mathrm{m}$ IRAS data by the Air Force Phillips Laboratory and Mission Research Corporation. Alternatively, standard four-band HIRES images can be requested from IPAC.

The original HIRES algorithm which produces highresolution IRAS images and later enhancements are described in Sec. 3. The MCM algorithm (Aumann et al. 1990) produces high-resolution images from the survey and additional observation (AO) data, using a nonlinear iterative scheme. The resulting images have resolution of about $1^{\prime}$, compared to the $4^{\prime}-5^{\prime}$ subtended by the $100 \mu \mathrm{m}$ band detectors in the IRAS focal plane. A description of the basic MCM algorithm is outlined in Sec. 3.1. In Secs. 3.2 and 3.4 we offer descriptions of artifact reduction algorithms, namely, using estimates of gain offset to eliminate striping, and using a Burg entropy metric in the iterative algorithm to suppress ringing around bright point sources. Detector data calibration and zodiacal light subtraction are carried out with a spin-off method of the destriping algorithm (Sec. 3.3). Validation of the algorithmic enhancements and output image properties is given in Sec. 4.

In the parallel processing each $1^{\circ} \times 1^{\circ}$ image field is mapped to an 8- or 16-node process grid, which shares the computation by loading different observation scans. An efficiency of $60 \%$ is reached with eight nodes. The parallelization strategy and pipeline implementation (which coordinates computation and data transfer on workstations and supercomputers) will be discussed in Sec. 5 .

\section{RELEVANT INFORMATION ABOUT IRAS}

The IRAS survey was designed for the identification of point sources, rather than as an imaging instrument. The data were taken with rectangular detectors that scanned the sky multiple times in "push-broom" fashion (e.g., see Fig. 1). The satellite data are fundamentally in the form of onedimensional data streams for each detector. During postprocessing it was discovered that two-dimensional images could be made by stitching together, i.e., coadding, these one-dimensional detector streams. This basic processing accounts for many of the characteristics of the IRAS images. For example, stripes are a common image artifact because there are offset and gain variations in the one-dimensional detector streams. Also, the shape of the beam varies from place to place because the coverage (i.e., number and orien-

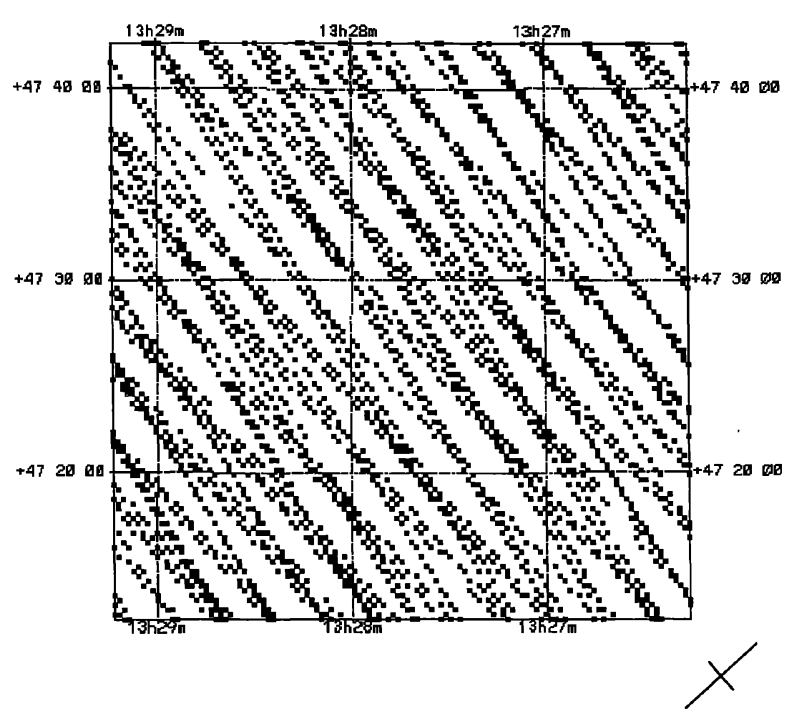

FIG. 1-IRAS scan pattern in M51. Dots represent $60 \mu \mathrm{m}$ detector footprint center positions. Lower right cross indicates FWHM of the $60 \mu \mathrm{m}$ detector response function.

tation of one-dimensional detector streams) is nonuniform. The effective data oversampling make the IRAS data amenable to resolution enhancement because of the geometric information contained within overlapping data samples.

The IRAS focal plane (shown in Fig. 2) included eight staggered linear arrays subtending $30^{\prime}$ in width, two in each of four spectral bands at $12,25,60$, and $100 \mu \mathrm{m}$. Data rate considerations forced the detector sizes to be much larger

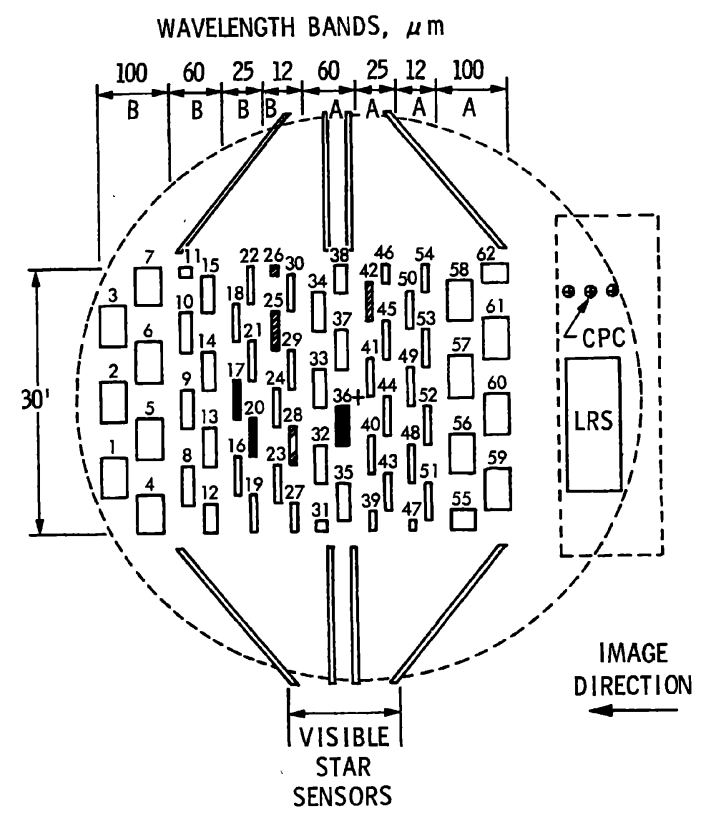

FIG. 2-A schematic drawing of the IRAS focal plane. The numbered rectangles in the central portion each represent the field of view of a detector, filter and field lens combination. The filled-in detectors were inoperative while the cross-hatched detectors showed degraded performance during the mission. 
TABLE 1

Definition of Notations

\begin{tabular}{cc}
\hline \hline$D_{i}$ & measured detector flux at data sample $i$ \\
$f_{j}$ & intensity at image pixel $j$ \\
$f_{j}^{(k)}$ & estimate of $f_{j}$ at iteration $k$ \\
$F_{i}=\sum_{j} r_{i j} f_{j}$ & mock data flux at sample $i$ given image $f_{j}$ \\
$C_{i}=D_{i} / F_{i}$ & detector correction factor \\
$c_{j}$ & pixel-correction factor \\
$G_{L}$ & gain offset of detectors in scanline $L$ \\
$D_{i}^{*}=G_{L} D_{i}$ & gain compensated detector flux \\
$C_{i}^{*}=D_{i}^{*} / F_{i}$ & gain compensated detector correction factor \\
$\Delta S$ & Burg entropy difference between two images $f_{j}$ and \\
$g_{j j^{\prime}}$ & $f_{j}+\Delta f_{j}$ \\
\hline \hline
\end{tabular}

than the diffraction limit of the telescope. The typical detector sizes were $45 \times 267,45 \times 279,90 \times 285$, and $180 \times 303$ arcsec (full width at half maximum response, FWHM), respectively, at the four wavelength bands.

This combination of focal plane, detector size, and scan pattern optimized detection of point sources in areas of the sky where the separation between sources was large compared to the sizes of the detectors. However, it complicates the construction of images of regions containing spatial structure on the scale of arcminutes.

\section{ALGORITHM}

A typical HIRES processing consists of the following steps:

(1) uncompressing and extracting calibrated data scans from archive;

(2) data preprocessing, including cross-scan offset calibration, baseline removal, deglitching, ${ }^{1}$ and noise estimation;

(3) subtraction of zodiacal emission (optional);

(4) reprojecting data to desired geometry (optional);

(5) high-resolution image reconstruction.

This section describes the algorithmic aspects of HIRES, specifically, issues involved in steps (2), (3), and (5). For details on the technical aspects of the implementation, see Sec. 5 .

Table 1 summarizes the notations used in this section.

\subsection{The Maximum Correlation Method}

Starting from a model of the sky flux distribution, the HIRES MCM algorithm folds the model through the IRAS detector responses, compares the result track by track ${ }^{2}$ to the observed flux, and calculates corrections to the model. One important characteristic is that the standard MCM algorithm conserves flux. The process is taken through about 20 iterations at which point artifacts limit further improvement. The algorithm yields a resolution of approximately $1^{\prime}$ at 60

${ }^{1}$ Deglitching stands for the removal of spurious non-source-like signals called glitches, typically caused by cosmic-ray events in individual detectors.

${ }^{2}$ Track, also called leg or scanline, refers to the set of data samples collected consecutively by one detector moving across a given field. $\mu \mathrm{m}$. This represents an improvement in resolution by as much as a factor of 20 in solid angle over the previous images from the IRAS Full Resolution Survey Coadder (FRESCO). We give a brief description of the MCM algorithm following the formalism and notations of Aumann et al. (1990).

Given an image grid $f_{j}$, with $n$ pixels $j=1, \ldots, n$, and $m$ detector samples (footprints) with fluxes $D_{i}: i=1, \ldots, m$, whose centers are contained in the image grid, an image can be constructed iteratively from a zeroth estimate of the image, $f_{j}^{0}=$ const. $>0$, for all $j$. In other words the initial guess is a uniform, flat, and positive definite map. For each footprint, a correction factor $C_{i}$ is computed as

$$
C_{i}=D_{i} / F_{i}
$$

where

$$
F_{i}=\sum_{j} r_{i j} f_{j}
$$

and $r_{i j}$ is the value of the $i$ th footprint's response function at image pixels $f_{j}$. Therefore $F_{i}$ is the current estimate of the $i$ th footprint's flux, given image grid $f_{j}$.

A mean correction factor for the $j$ th image pixel is computed by projecting the correction factor for the footprints into the image domain:

$$
c_{j}=\left[\sum_{i}\left(r_{i j} / \sigma_{i}^{2}\right) C_{i}\right] /\left[\sum_{i}\left(r_{i j} / \sigma_{i}^{2}\right)\right] .
$$

The weight attached to the $i$ th correction factor for the $j$ th pixel is $r_{i j} / \sigma_{i}^{2}$, where $\sigma_{i}$ is the $a$ priori noise assigned to the $i$ th footprint.

The $k$ th estimate of the image is computed by

$$
f_{j}^{(k)}=f_{j}^{(k-1)} c_{j}
$$

In practice when the footprint noise $\sigma_{i}$ is not easily estimated, an equal noise value for all footprints is assumed, and the MCM is identical to the Richardson-Lucy algorithm (Richardson 1972; Lucy 1974).

\subsection{Destriping Algorithm}

Stripes are the most prominent artifacts of the HIRES images. HIRES takes in the IRAS detector data, and if not perfectly calibrated, would try to fit the gain differences in the detectors by a striped image. The striping builds up in amplitude and sharpness along with the HIRES iterations, as the algorithm refines the "resolution" of the stripes [see Figs. 3(a) and 3(b)].

The IPAC program LAUNDR (Fowler and Melnyk 1990) invokes several one-dimensional flat fielding and deglitching techniques. The basic algorithm applied is clamping the background of different scan lines (taken as a low percentile in detector flux histogram for each scan line) to a common level. For the purpose of destriping, the one-dimensional algorithm works well for regions with a well-defined baseline, but the result is not satisfactory for regions where structure exists in all spatial frequencies. 


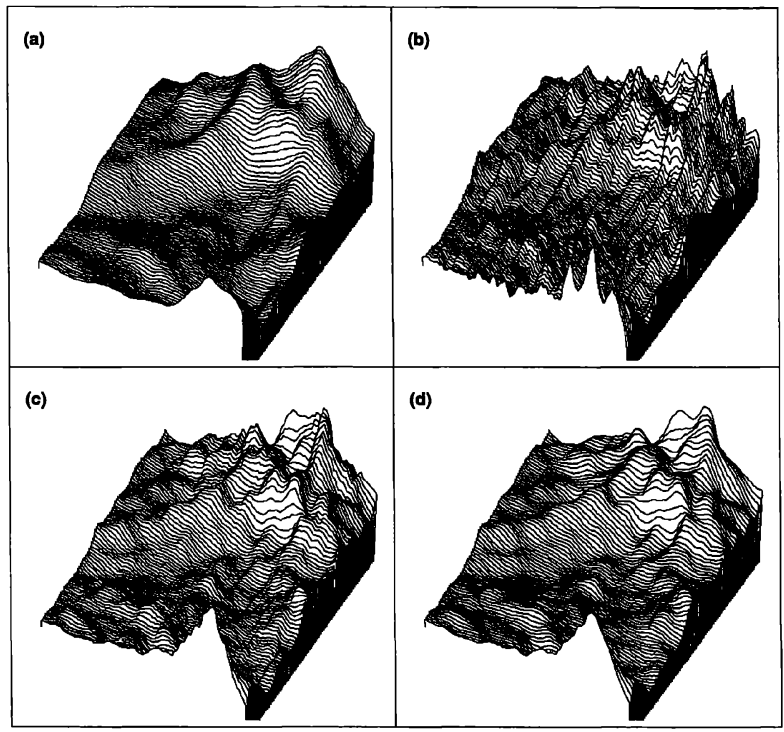

FIG. 3-(a) 1st iteration image for a field in $\rho$ Ophiuchi (100 $\mu \mathrm{m}$ band); (b) 20th iteration, standard HIRES; (c) 20th iteration, with uniform gain compensation; (d) 20th iteration, with local gain compensation. Size of image is $1^{\circ} \times 1^{\circ}$. Height of surface represents flux. Local gain compensation method produces high-resolution images that are free of stripes, the most common artifact in standard HIRES processing.

\subsubsection{Destriping with Uniform Gain Compensation}

Our approach combines the image reconstruction and the destriping process. Since the striping gets amplified through the iterations, the idea of applying constraints to the correction factors is natural.

Assume footprints in the same leg $L$ suffer from the same unknown gain offset $G_{L}$; then

$$
D_{i}^{*}=G_{L} D_{i}
$$

is the "true" detector flux, had the detector gain been perfectly calibrated. The $G_{L}$ 's can be seen as extra parameters to be estimated, besides the image pixels $f_{j}$. Under a Poisson framework, the maximum-likelihood estimate for $G_{L}$ is

$$
\prod_{i \text { in } \operatorname{leg} L}\left(\frac{G_{L} D_{i}}{F_{i}}\right)^{D_{i}}=\prod_{i \text { in leg } L}\left(C_{i}^{*}\right)^{D_{i}=1},
$$

in which $C_{i}^{*}$ is the gain compensated correction factor. $C_{i}^{*}$ is then used in place of $C_{i}$ in Eq. (3) to compute the pixel correction factors. A new set of $G_{L}$ is estimated for every MCM iteration.

This choice of the unknown gain offset $G_{L}$ minimizes the mutual information between the sets $D_{i}^{*}$ and $F_{i}$ in the leg, i.e., the resulting correction factors $C_{i}^{*}$ will extract the minimum amount of information from the stream $D_{i}^{*}$. From the viewpoint of the maximum entropy principle, this is the most reasonable choice.

From another point of view, this strategy works because the procedure of averaging $C_{i}$ 's to get $c_{j}$ has a smoothing effect on the image, so that the image $f_{j}$ and estimated flux $F_{i}$ do not contain as much striping power as the footprints $D_{i}$.

\subsubsection{Destriping with Local Gain Compensation}

A further complication lies in the fact that the assumption of a uniform gain offset in a certain leg is only approximately true. Various hysteresis effects (e.g., see Chap. IV of IRAS Catalogs and Atlases: Explanatory Supplement 1988) cause the gain to drift slightly within the $1^{\circ}$ range. A more aggressive form of the destriping algorithm estimates the gain offset locally as the weighted geometric mean of the correction factors for nearby footprints, so the estimated gain correction for each footprint varies slowly along the leg. The local gain offset is compared to the global one estimated from the entire leg, and if they differ by more than $10 \%$ then the global value is used, since the gain is not expected to drift that much over a $1^{\circ}$ scale, and the variation in computed offset average is most likely due to real local structure. We used an averaging length of $10^{\prime}$ to estimate the local offset. Because it is larger than the spatial resolution of the first iteration image $\left(5^{\prime}\right)$, it is safe to refer the average correction factor on that scale as due to gain offset. The $10^{\prime}$ length scale is also small enough to capture the drifting behavior of the gain, as shown by visual inspection of both the output images and their Fourier power spectra. Unlike the standard HIRES algorithm (in which stripes are amplified throughout the iterations), the local gain compensation decreases the striping power monotonically to a negligible level after roughly ten iterations.

One aspect of the local gain compensation method is that the computed correction factors can cause the flux scale to drift slightly. This is solved by requiring an occasional iteration using the standard MCM algorithm to enforce flux conservation. In practice a standard MCM iteration performed at 10 and 20 iterations produced no noticeable re-introduction of stripes.

\subsubsection{Results of the Destriping Algorithm}

Figure 3 demonstrates the striking effect of the destriping algorithm. Figure 3(a) shows the first iteration (FRESCO) image for a $1^{\circ} \times 1^{\circ}$ field in $\rho$ Ophiuchi, which is smooth (blurry). Figure $3(\mathrm{~b})$ is the 20th iteration image of the field obtained with the standard HIRES algorithm, and is contaminated with strong striping artifacts. A tremendous improvement is seen in Fig. 3(c) which is produced with uniform gain compensation, although some weak stripes are still visible. Finally, using the local gain compensation method gives a stripe-free image, Fig. 3(d). It is also apparent that Fig. 3(d) contains many high spatial frequency features that are absent in Fig. 3(a).

\subsection{Subtraction of Zodiacal Emission}

Zodiacal dust emission is a prominent source of diffuse emission in the IRAS survey, especially in the 12 and 25 $\mu \mathrm{m}$ bands. The zodiacal contribution to the observed surface brightness depends on the amount of interplanetary dust along the particular line of sight, an amount which varies with the Earth's position within the dust cloud. Consequently, the sky brightness of a particular location on the sky, as observed by IRAS, changes with time as the Earth moves along its orbit around the Sun. The different zodiacal 
emission levels in different scanlines, if not subtracted, can cause step discontinuities in the images if adjacent patches of sky were observed at different times. Cross-scan destriping helps bring together the background level of scanlines passing through the same local field at different times, but for large-scale astronomical studies it is essential to have the zodiacal emission removed.

A physical model of the zodiacal foreground emission based on the radiative properties and spatial distribution of the zodiacal dust was developed by Good (1994). The IRAS Sky Survey Atlas (ISSA; Wheelock et al. 1994) made use of this model and subtracted the predicted zodiacal emission from the detector data before coadding them. The resulting ISSA images show a strong Galactic background at 60 and $100 \mu \mathrm{m}$ that is associated with molecular and $\mathrm{H}$ I clouds in the Galaxy (e.g., Weiland et al. 1986; Terebey and Fich 1986; Boulanger and Perault 1988; Sodroski et al. 1989; Scoville and Good 1989). It is desirable for the highresolution IRAS Galaxy Atlas to be sensitive to this largescale component of Galactic emission.

The zodiacal subtraction is a fairly complicated process, and since HIRES has typically been used for small fields only, the zodiacal emission has been historically treated as part of the local background that is subtracted (and thrown away) during the LAUNDR step. Fortunately the destriping algorithm offers a way to make use of the zodiacal subtraction effort that went into the ISSA images-we can estimate the zodiacal emission by comparing input detector data and simulated data from the ISSA images, ${ }^{3}$ and calibrate the input data to obtain a background level that is consistent with the ISSA images. The zodiacal emission is taken as a median difference of flux between real and simulated data, computed over a 1 degree range (the characteristic scale of zodiacal foreground variation), and is then subtracted from the real data. The method therefore only affects the low spatial frequency component of the data, and does not disturb the small-scale signals.

When the input ISSA image contains significant striping (at width around $7^{\prime}$, that is, the distance of neighboring scan tracks, a much larger scale than the HIRES stripes), it is necessary to first smooth the ISSA image with a large kernel $\left(15^{\prime}\right)$ before doing the zodiacal subtraction. Otherwise the calibrated detector data would retain the large distance scale offsets, and the gain compensation destriping described in Sec. 3.2 would not be able to estimate the gain variations correctly and would leave the wide stripes at different flux levels.

For validation of this procedure see the comparison of surface brightness (output HIRES vs. ISSA) described in Sec. 4.4 .

\subsection{De-ringing Algorithm}

For many image reconstruction algorithms, ringing artifacts (or "ripples") appear when a bright point source exists over a nonzero background. The mechanism of the artifact

${ }^{3}$ The ISSA images were corrected to the same flux calibration scale as HIRES (see Sec. 4.4), before calculating the simulated data. can be understood as the Gibbs phenomenon (a sharp cutoff in high spatial frequency signal incurs ripples in the position domain). Numerous approaches have been taken to reduce this kind of artifact, such as that of regularizing operator in the linear restoration regime (e.g., Zervakis and Venetsanopoulos 1992), and maximum entropy methods (Press et al. 1992).

A variant of the log entropy MART ${ }^{4}$ (De Pierro 1991),

$$
f_{j}^{(k)}=f_{j}^{(k-1)}+\left(f_{j}^{(k-1)}\right)^{2} \sum_{i} \frac{r_{i j}}{F_{i}^{2}}\left(D_{i}-F_{i}\right)
$$

was tested on IRAS data.

The $\left(f_{j}^{(k-1)}\right)^{2}$ factor in the correction term indicates a Burg entropy metric in the image space: the entropy loss (Burg) involved in changing one's knowledge from $f_{j}$ to $f_{j}+\Delta f_{j}$ is

$$
-\Delta S=\sum_{j} \frac{f_{j}+\Delta f_{j}}{f_{j}}-1-\log \frac{f_{j}+\Delta f_{j}}{f_{j}}=\sum_{j} \frac{1}{f_{j}^{2}}\left(\Delta f_{j}\right)^{2}
$$

for small $\Delta f_{j}$, so if we define the distance as $\sqrt{|\Delta S|}$, the metric tensor becomes

$$
g_{j j^{\prime}}= \begin{cases}1 / f_{j}^{2}, & \text { if } j=j^{\prime}, \\ 0, & \text { otherwise. }\end{cases}
$$

The $f_{j}^{2}$ factor then acts to change the covariant gradient vector to contravariant. (See Skilling, 1986, for a similar analysis for the Shannon entropy.)

The Burg entropy metric effectively boosts the correction factor for brighter pixels, so the bright point source is fitted better in the earlier iterations, which circumvents the corruption of background propagated from the misfit near the point source.

The prior knowledge signified by using maximum Burg entropy estimation rule has been discussed in Jaynes (1986) and Frieden (1985). According to Frieden, the class of optical objects described by the Burg entropy prior would tend to consist of a relatively small number of randomly placed bright cells, the rest being dim, befitting the bright point source scene we are concerned with.

Suppression of ringing may potentially lead to better photometry determination of the point source through better background determination, and helps solve source confusion problems, which are especially prominent in the Galactic plane.

Although the above algorithm gave satisfactory results for some test fields (e.g., see Fig. 4), it suffers from several problems. First, boosting the correction factors for brighter pixels biases the total flux toward a higher value, and when this is combined with the destriping algorithm, which essentially is a self-calibrating scheme, gives rise to bootstrapping and uncontrolled growth of flux in the image. This problem can be solved by performing a standard MCM iteration with no destriping and de-ringing applied, before writing out the image. Richardson-Lucy's good property of local flux conservation thus brings back the image flux to the correct level.

${ }^{4}$ Multiplicative algebraic reconstruction technique. 


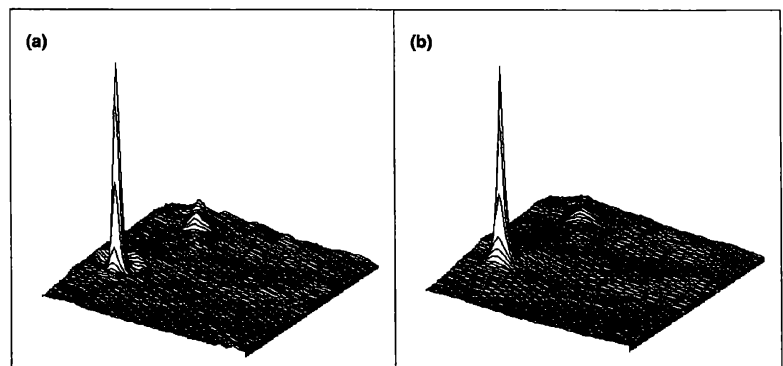

FIG. 4 (a) Point source I 16293-2422 in $\rho$ Ophiuchi, no ringing suppression. (b) Same field, using entropy prior for ringing suppression. Size of image is $1^{\circ} \times 1^{\circ}$. Peak flux in (a) is $3749 \mathrm{MJy} \mathrm{sr}^{-1}$, and $3329 \mathrm{MJy} \mathrm{sr}^{-1}$ in (b).

Another more serious problem lies more deeply at the heart of the Burg iterative scheme. Since the correction for fainter pixels is damped near bright ones, the Burg iteration is slower at trimming the lobes of point sources. In addition, convergence of a faint source near a bright one is also suppressed, along with the formation of the ring.

For these reasons further research is needed to understand the behavior of the ringing suppression algorithm, before it can be incorporated into the production algorithm. In addition, we are investigating an adaptive data splitting method which aims to separate background and point-source fluxes during the reconstruction. The image space reconstruction algorithm (ISRA, e.g., De Pierro 1991) is also being studied for its potential in ringing suppression.

\section{OUTPUT VALIDATION}

This section discusses the verification of output image properties.

To test the authenticity of high resolution features produced by the MCM algorithm, Aumann et al. (1990) compared the $60 \mu \mathrm{m}$ HIRES image of M101 with the IRAS Point Source Catalog (1988) and previously known H II regions (based on observations at ultraviolet, infrared, and radio wavelengths). Also Rice (1993) examined the structural reliability of HIRES maps for three test galaxies, M51, M33, and NGC 6822, using the following truth tables: (1) a farinfrared KAO map of M51, (2) optical light photographic images of the three test galaxies, (3) four additional types of "high-resolution" maps constructed from independent IRAS data, and (4) a simulated map of the radio emission of bright $\mathrm{H}$ II regions in M33 constructed from a catalog of $20 \mathrm{~cm}$ radio continuum sources in the galaxy.

We have compared the gain compensation destriped images with the original HIRES images for the above fields (and numerous others), and have found good agreement in the reconstructed features (except for the lack of striping). The following sections deal with validation of gain offset recovery, source photometry, source positions, and surface brightness.

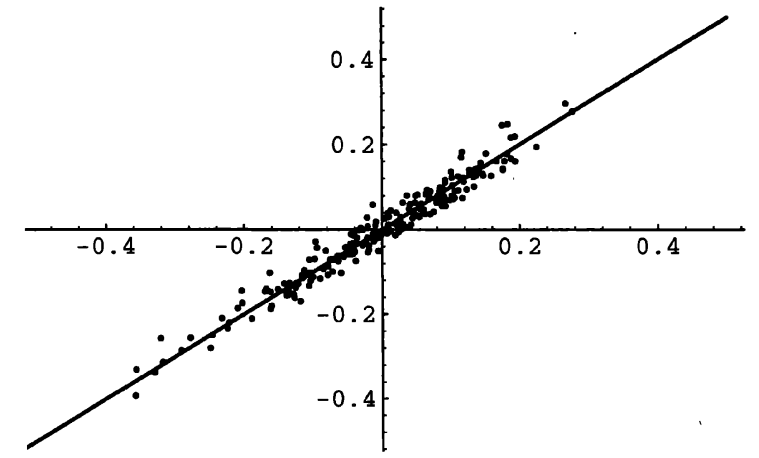

FIG. 5-Recovery of artificially introduced offsets. Vertical: log of recovered gain offset; horizontal: log of introduced gain offset.

\subsection{Validation of Gain Offset Recovery}

To verify the gain offset estimation, a test to recover artificially introduced offsets was carried out. A stripe-free image was used to generate a set of simulated detector data, and Gaussian-generated gain offsets were applied to the legs. This set of data was fed to the uniform gain offset compensation program, and a scatter plot of the recovered versus introduced offsets is shown in Fig. 5.

The introduced offsets are Gaussian with standard deviation 0.12. The standard deviation of residual offset after compensating for estimated offset is $\mathbf{0 . 0 2 4}$, indicating a factor-of- 5 reduction in striping amplitude ( 25 in power).

The reconstructed image is stripe-free and visually indistinguishable from the input image. This suggests that the uniform gain compensation is capable of its designed goals, and that the residual striping seen in the real data [Fig. 3(c)] is in fact due to small gain yariations within the legs, lending support to the local gain compensation method.

\subsection{Validation of Source Photometry}

To verify the photometric integrity of HIRES images using gain compensation destriping, detector data processed with LAUNDR for the sources M51, M101, and $\beta$ Pictoris were fed to the gain compensation algorithm and source fluxes at the 20th iteration were compared with results from the standard MCM algorithm. The source fluxes were determined using aperture photometry, by calculating the background level as the median of pixel fluxes in an annulus around the source, and subtracting the background from the total flux within the circle. The maximum percentage difference between the two sets of results is $5.4 \%$ (see Table 2 for the comparison).

\subsection{Validation of Source Positions}

To validate the reprojection code and source positions in the output HIRES images, 39 sources near the Galactic plane were checked against the IRAS Point Source Catalog (1988). A total of 39 sources between Galactic latitude $-1.7^{\circ}$ and $1.7^{\circ}$ ( $\times$ two bands) were tested in these longitude intervals: $119^{\circ}-129^{\circ}, 215^{\circ}-223^{\circ}$, and $355^{\circ}-2^{\circ}$, with 21,8 , and 10 
TABLE 2

Comparison of Source Photometry (Destriping vs Non-Destriping)

\begin{tabular}{|c|c|c|c|c|c|c|}
\hline Source & Wavelength $(\mu \mathrm{m})$ & Preprocessing & Destripe? & 1st (Jy) & 20th (Jy) & Diff. at 20th iter. (\%) \\
\hline M51 & 60 & $b^{a}$ & no & 127.30 & 131.24 & - \\
\hline M51 & 60 & $\mathrm{br}$ & yes & 125.53 & 126.63 & -3.5 \\
\hline M51 & 60 & do & no & 127.23 & 130.98 & - \\
\hline M51 & 60 & do & yes & 127.23 & 130.25 & -0.6 \\
\hline M51 & 100 & br & no & 286.64 & 303.80 & - \\
\hline M51 & 100 & $\mathrm{br}$ & yes & 283.28 & 295.11 & -2.9 \\
\hline M51 & 100 & do & no & 283.86 & 299.79 & - \\
\hline M51 & 100 & do & yes & 283.86 & 296.05 & -1.2 \\
\hline M101 & 60 & $\mathrm{br}$ & no & 85.30 & 85.34 & - \\
\hline M101 & 60 & $\mathrm{br}$ & yes & 81.72 & 81.76 & -4.2 \\
\hline M101 & 60 & do & no & 83.05 & 84.41 & - \\
\hline M101 & 60 & do & yes & 83.08 & 84.43 & +0.0 \\
\hline M101 & 100 & $\mathrm{br}$ & no & 210.95 & 217.56 & - \\
\hline M101 & 100 & $\mathrm{br}$ & yes & 207.92 & 209.97 & -3.5 \\
\hline M101 & 100 & do & no & 210.70 & 216.71 & - \\
\hline M101 & 100 & do & yes & 210.71 & 214.37 & -1.1 \\
\hline$\beta$ Pictoris & 60 & $\mathrm{br}$ & no & 22.03 & 21.84 & - \\
\hline$\beta$ Pictoris & 60 & br & yes & 22.10 & 20.96 & -4.0 \\
\hline$\beta$ Pictoris & 60 & do & no & 21.58 & 20.96 & - \\
\hline$\beta$ Pictoris & 60 & do & yes & 21.51 & 21.38 & +2.0 \\
\hline$\beta$ Pictoris & 100 & br & no & 10.19 & 10.95 & - \\
\hline$\beta$ Pictoris & 100 & $\mathrm{br}$ & yes & 11.96 & 10.86 & -0.8 \\
\hline$\beta$ Pictoris & 100 & do & no & 10.45 & 9.90 & - \\
\hline$\beta$ Pictoris & 100 & do & yes & 10.42 & 10.43 & +5.4 \\
\hline
\end{tabular}

${ }^{\mathrm{a}} \mathrm{br}=\mathrm{b}$ aseline removal; do=destripe only, referring to the cross-scan offset done in LAUNDR (not to be confused with gain compensation destriping).

sources in each interval, respectively. All sources had fluxes $>1 \mathrm{Jy}$ at 60 and $100 \mu \mathrm{m}$. Sources lying within 10 arcmin of each other were excluded. Also some sources close to the Galactic center were excluded, due to a large gradient in the background intensity. For each chosen source, a circular area with radius $5^{\prime}$ was defined (centered at the PSC position), and the area's flux-weighted centroid was taken as the HIRES point source position and compared against the PSC position. For $60 \mu \mathrm{m}$ band, the distances between HIRES position and PSC position have an average of $9.3^{\prime \prime}$ and standard deviation $4.9^{\prime \prime}$, and for $100 \mu \mathrm{m}, 8.3^{\prime \prime} \pm 4.4^{\prime \prime}$.

\subsection{Validation of Surface Brightness}

To test the surface brightness of zodiacal-subtracted HIRES images, they were rebinned to ISSA geometry (using boxcar averaging) and compared pixel by pixel against the ISSA images.

Multiplicative flux scale (ac/dc) correction was applied to the ISSA images before calculating the surface brightness correlation. The IRAS detectors had a dwell-time-dependent responsivity change. Hence, the gain changes as a function of source size: at the IRAS survey speed of $3.85 \operatorname{arcmin~}^{-1}$, the gains leveled off for structure on the order of $30^{\prime}$ in extent. Thus there are two calibrations for the IRAS data, the calibration appropriate for point sources, known as the ac calibration, and the calibration appropriate to very extended structures, known as the dc calibration. To convert fluxes and surface brightness measured from dc-calibrated products to the ac (same as the Point Source Catalog) calibration, the values must be divided by $0.78,0.82,0.92$, and 1.0 at 12,25 , 60 , and $100 \mu \mathrm{m}$, respectively. HIRES uses the ac calibration, while the ISSA images are on the dc scale (see Chap. VI of IRAS Catalogs and Atlases: Explanatory Supplement 1988).

The standard deviation of the pixel-by-pixel $\left(1.5^{\prime}\right)$ difference is less than $6 \%$ for the first iteration HIRES vs. ISSA, and less than $12 \%$ for the 20th iteration HIRES vs. ISSA (Table 3). The difference is larger at the 20th iteration as the rebinned HIRES images are still sharper than ISSA, while the first iteration HIRES images have a resolution similar to the $4^{\prime}$ to $5^{\prime}$ of ISSA.

No systematic offset was found between HIRES and ISSA surface brightness after applying the ac/dc correction (HIRES surface brightness should be multiplied by 0.92 and 1.0 at 60 and $100 \mu \mathrm{m}$, respectively, to be consistent with ISSA). Typical scatter plots of $\log$ HIRES/ISSA vs. ISSA intensities are shown in Fig. 6.

TABLE 3

Comparison of Surface Brightness

\begin{tabular}{lccr}
\hline \hline Position & Wavelength $(\mu \mathrm{m})$ & 1st vs. ISSA & 20th vs. ISSA \\
\hline G000.5,+0.5 & 60 & $0.024 \pm 0.064$ & $0.013 \pm 0.100$ \\
G121.5,+0.5 & 60 & $0.017 \pm 0.035$ & $0.008 \pm 0.112$ \\
G126.5,-0.5 & 60 & $0.007 \pm 0.040$ & $-0.010 \pm 0.119$ \\
G218.5,-0.5 & 60 & $0.006 \pm 0.033$ & $-0.002 \pm 0.080$ \\
G000.5, 0.5 & 100 & $0.031 \pm 0.057$ & $0.020 \pm 0.083$ \\
G121.5, 0.5 & 100 & $0.003 \pm 0.016$ & $-0.003 \pm 0.068$ \\
G126.5,-0.5 & 100 & $0.008 \pm 0.028$ & $-0.004 \pm 0.081$ \\
G218.5,-0.5 & 100 & $0.005 \pm 0.016$ & $0.000 \pm 0.047$ \\
\hline \hline
\end{tabular}

${ }^{a}$ Comparison was done for $1^{\circ}$ radius circles centered at positions shown in first column. Differences are represented by mean \pm standard deviation of the quantity $\log ($ HIRES/ISSA). ISSA images were corrected to ac scale before comparison. 
(a)

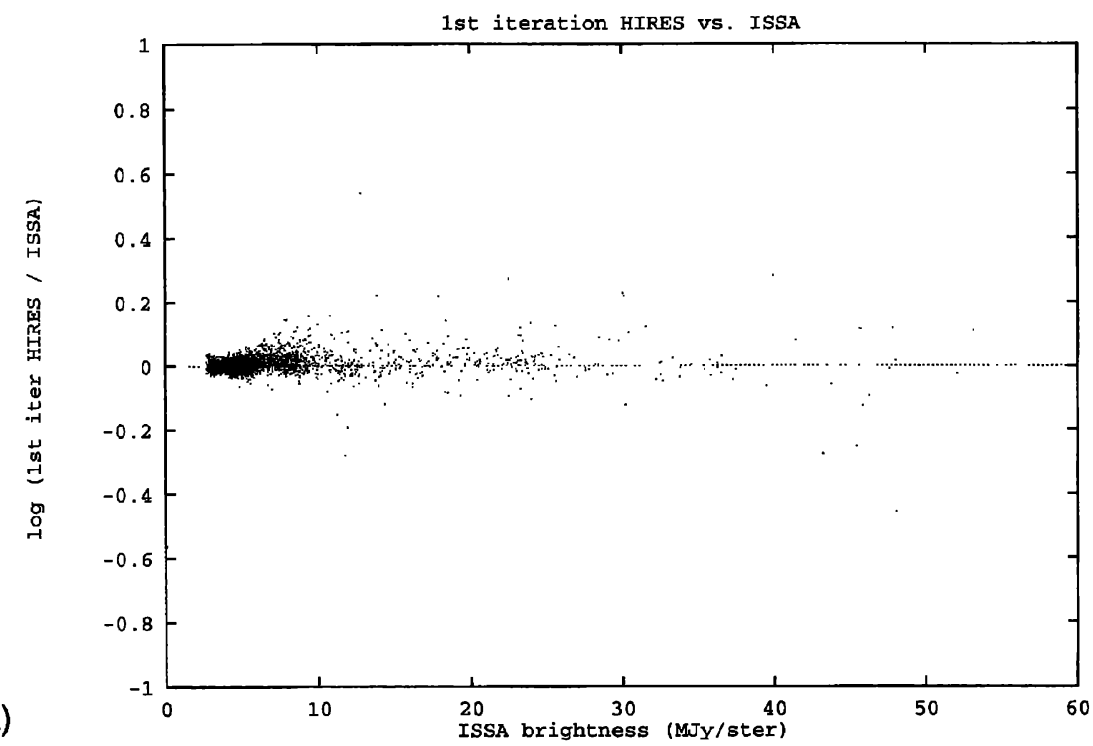

(b)

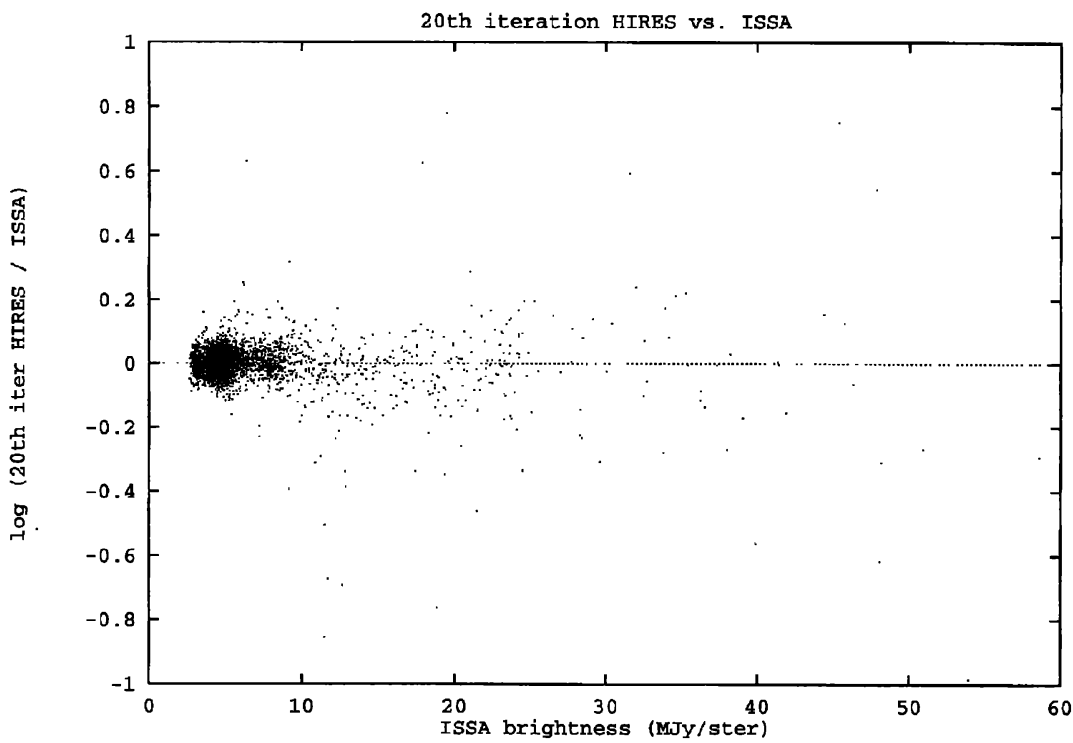

FIG. 6 - Comparison of HIRES and ISSA Surface Brightness. $1^{\circ}$ radius circular area centered at G218.5, -0.5 (60 $\left.\mu \mathrm{m}\right)$ were compared. Top: comparison of 1st iteration HIRES vs. ISSA; bottom: comparison of 20th iteration HIRES vs. ISSA. Vertical: log of HIRES/ISSA; horizontal: ISSA intensity in MJy sr ${ }^{-1}$. ISSA image was corrected to ac scale before comparison.

\section{IMPLEMENTATION}

This section gives a detailed account of the technical aspects of a pipeline consisting of coordinated processing on workstations and parallel supercomputers, which produces HIRES images in mass quantities (see Fig. 7).

\subsection{Overview of the Production Pipeline}

IRAS detector data, known as CRDD (calibrated, reconstructed detector data), grouped in $7^{\circ} \times 7^{\circ}$ plates, reside in the "Level 1 Archive." The first step in the pipeline for mass production of HIRES images is to extract data covering a specific field with "SnipScan" and feed them into LAUNDR for calibration and various other preprocessing.
We take the $7^{\circ} \times 7^{\circ}$ LAUNDRed plates and use the algorithm described in Sec. 3.3 to subtract the zodiacal background emission. This step requires the corresponding ISSA image as supplement input (SmLAUN in Fig. 7).

Following the calibration and zodiacal subtraction, the detector files are broken into $1.4^{\circ} \times 1.4^{\circ}$ fields, and reprojected into Galactic coordinates (from equatorial) if required, with field centers separated by $1^{\circ}$ (BrkDet in Fig. 7). The factorof- 2 overlap is a conservative insurance against discontinuity across field boundaries, as local destriping and different flux bias level will be applied to each small field. $1.4^{\circ} \times 1.4^{\circ}$ is also the maximal field size with complete coverage allowed within one Level 1 plate, given the 2 degree redundancy of the plates and arbitrary location and orientation of the small field relative to the Level 1 plate. 


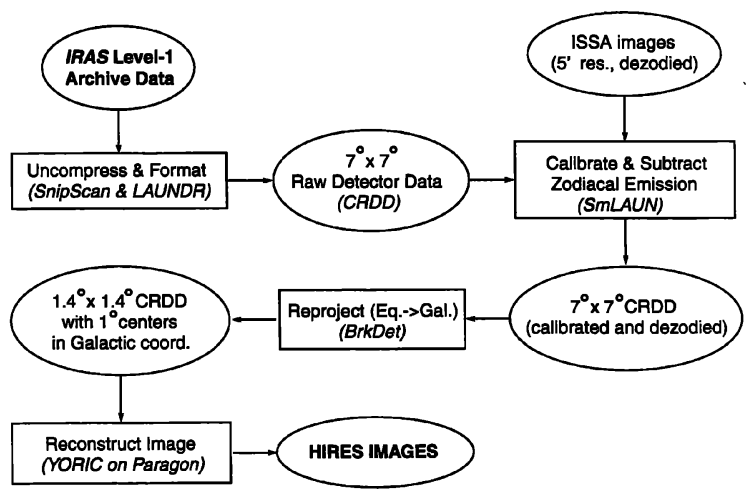

FIG. 7-Outline of the HIRES production pipeline.

All operations described above are carried out on workstations, and the total processing time for one $7^{\circ} \times 7^{\circ}$ plate, one wavelength band, averages to $80 \mathrm{~min}$ on a Sun SPARCstation 10 , most of which is spent on decompressing and extracting the data from the IRAS Level 1 Archive.

The small field $\left(1.4^{\circ} \times 1.4^{\circ}\right)$ detector files are then processed into HIRES images, which is done on the Intel Paragon supercomputer. The CPU time taken for this stage is about 100 node-hours ${ }^{5}$ for one wavelength band and one $7^{\circ} \times 7^{\circ}$ plate. The next section gives a detailed discussion for the parallelization of the maximum correlation method.

The output images are stored to UniTree, a high-capacity storage system on the Paragon using HIPPI interface. About 200 megabytes of output data (images and auxiliary maps) are generated for one bandplate.

\subsection{Parallelization}

A flow chart of one iteration of the parallelized program is shown in Fig. 8.

Profiling ${ }^{6}$ a typical HIRES process showed that more than 95\% of the total execution time was spent within the code which calculates the footprint and image correction factors (see Fig. 8). In the parallel decomposition of the problem, each processor takes care of footprints from a set of scanlines. The reasons for doing this are the following:

(1) Small programing effort. The essence of the original HIRES architecture is left untouched.

(2) Footprints in one leg share the same response function grid, except for a translation, which is basically the reason the original code processes the data one leg at a time. Keeping the whole leg in one processor is therefore a natural choice, which minimizes local memory usage.

(3) As we discussed in Sec. 3.2, IRAS detectors have gain differences which are especially prominent for the 60 and $100 \mu \mathrm{m}$ bands. The gain offset can be estimated from correction factors in the same leg, which came from the same detector.

${ }^{5}$ Number of node-hours $=$ number of computing nodes $\times$ number of hours of real processing time.

${ }^{6}$ Profiling stands for timing analysis of subroutines in the program.

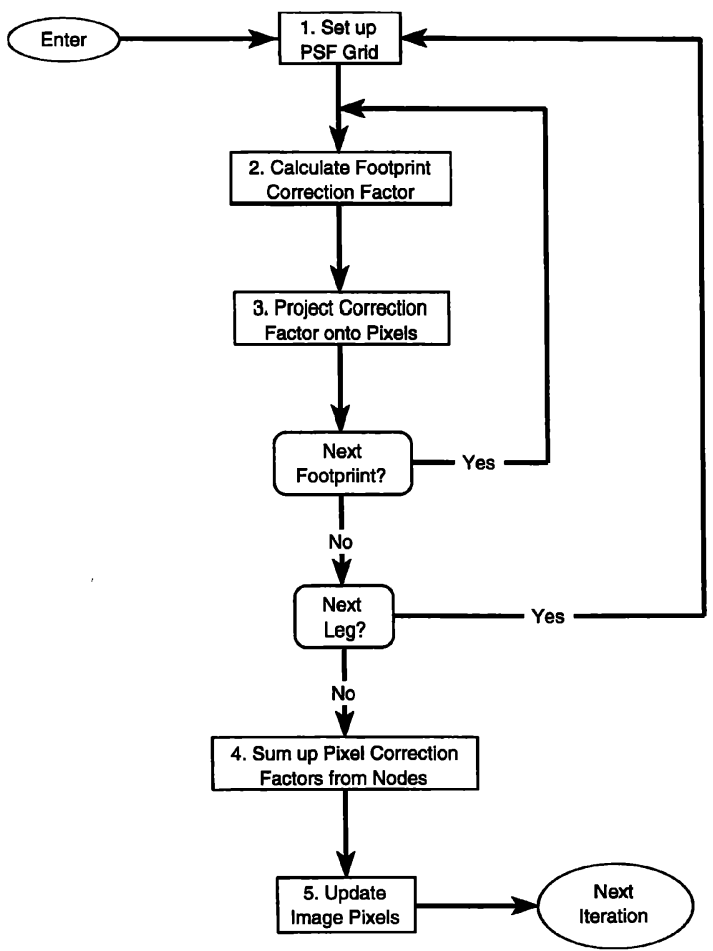

FIG. 8-Flow chart of one iteration in the parallel program.

Intermediate disk files for footprint data $\left(D_{i}\right)$ and response function grids $\left(r_{i j}\right)$ in the sequential program are replaced by arrays held in memory of the processors (step 1 in Fig. 8), for sake of easier programming and reduction in I/O. This is feasible in the parallel implementation as each processor now holds only a fraction of the entire data set.

Each node calculates the correction factor $C_{i}$ 's for its share of footprints (step 2), and projects them onto the pixels covered by the footprints (step 3). A global sum over all processors for the correction factor $c_{j}$ 's for each image pixel is performed at end of each iteration (step 4), and the weighted average is taken, which is then applied to the image pixel value (step 5).

Decomposition in the image domain was not carried out for the $1^{\circ} \times 1^{\circ}$ field, eliminating the need for ghost boundary communication, which would be significant and complicated to code, due to the large size and irregular shape of the detector response function. This helped maintaining the parallel code similar in structure to the sequential one, making simultaneous upgrades relatively easy.

The efficiency of the parallel program depends on the scan coverage of the field processed. The computation time is roughly proportional to the total coverage (i.e., total number of footprints), while the communication overhead is not related to footprints and is only dependent upon the image array size. So the efficiency is higher for a field with higher coverage.

For a large field (e.g., $\left.7^{\circ} \times 7^{\circ}\right)$, the detector measurements are broken into $1.4^{\circ} \times 1.4^{\circ}$ pieces with $0.4^{\circ}$ overlap. Each $1.4^{\circ} \times 1.4^{\circ}$ field is loaded on to a subgroup of 8 or 16 processors. The overlap was chosen conservatively so that crop- 
TABLE 4

Speed Comparisons for $60 \mu \mathrm{m}$ Band of M51

\begin{tabular}{ll}
\hline \hline Sun SPARCstation 2 & $720 \mathrm{~s}$ \\
Single node of the Paragon & $640 \mathrm{~s}$ \\
Eight nodes of the Paragon & $137 \mathrm{~s}$ \\
\hline \hline
\end{tabular}

ping the overlap after HIRES ensures smoothness at the boundaries. Mosaicked images made from adjacent fields turn out to be seamless to the human eye.

Currently the parallel program runs on a 512-processor Intel Paragon using Intel's NX communication routines under the OSF/1 operating system. It also runs on the SandiaUNM Operating System along with the provided communication library (SUNMOS; Maccabe et al. 1993), which is available on Paragon and nCUBE, and provides significant performance increase.

The output images from the parallel computers are compared with those from the standard HIRES program running on a Sun SPARCstation. The differences are well within the range of numerical round-off errors. At the 20th iteration, the standard deviation of (NewImage-OldImage)/OldImage averages to about $10^{-4}$.

The global sum operation, which collects pixel correction factors from different nodes, is the primary source of communication overhead in the parallel program.

The executable code was compiled and linked with a math library conformant to the IEEE 754 standard, and the compiler options were fine-tuned to give the best execution speed. For the $60 \mu \mathrm{m}$ band of M51 (baseline removed data), a time comparison is shown in Table 4.

A speed increase of about 7 times is achieved with 16 processors and 5 times with 8 processors for a $1^{\circ} \times 1^{\circ}$ field. Equivalently a 64 square degree field can be processed using 512 nodes, with a speedup factor of 320 . For production runs on the Paragon, we customarily use 128 nodes to process 16 small fields simultaneously. Each bandplate would therefore take roughly $1.5 \mathrm{hr}$ of real time. Various scripts are used to automate the data transfer and program launching.

\section{SUMMARY}

The parallelization and algorithmic enhancements of the IPAC HIRES program have been described. These efforts have enabled production of HIRES images by IPAC using the Intel Paragon supercomputer.

We are now in the process of producing the IRAS Galaxy Atlas, a complete atlas of the Galactic plane $\left( \pm 5^{\circ}\right.$ latitude) at 60 and $100 \mu \mathrm{m}$ with arcminute resolution, as well as maps of the Orion, Ophiuchus, and Taurus-Auriga clouds complexes.

We thank Tom Soifer, Joe Mazzarella, and Jason Surace for their involvement and helpful suggestions during the project. We are grateful to George Aumann, John Fowler, and Michael Melnyk for developing the original HIRES program, especially John Fowler who helped with the port of HIRES to the Intel computers by explaining the structure and details of the program, and provided advice throughout the algorithmic developments. Thanks are also due to Ron Beck and Diane Engler who handled numerous HIRES processing requests and are running the IRAS Galaxy Atlas production. This research received support from the NASA Astrophysics Data Program under Contract No. NAS5-32642, and was performed in part using the Intel Touchstone Delta and the Intel Paragon operated by Caltech on behalf of the Concurrent Supercomputing Consortium.

\section{REFERENCES}

Aumann, H. H., Fowler, J. W., and Melnyk, M. 1990, AJ, 99, 1674 Beichman, C. A., Myers, P. C., Emerson, J. P., Harris, S., Mathieu, R., Benson, P. J., and Jennings, R. E. 1986, ApJ, 307, 337

Beichman, C. A. 1987, ARAA, 25, 521

Bontekoe, T. R., Koper, E., and Kester, D. J. M. 1994, A\&A, 284, 1037

Boulanger, F. B., and Perault, M. 1988, ApJ, 330, 964

Clemens, D. P., Yun, J. L., and Heyer, M. H. 1991, ApJS, 75, 877

Fowler, J. W., and Melnyk, M. 1990, LAUNDR Software Design Specifications (Pasadena, IPAC)

Frieden, B. R. 1985, in Maximum-Entropy and Bayesian Methods in Inverse Problems, ed. C. R. Smith and W. T. Grandy, Jr. (Dordrecht, Reidel), p. 133

Good, J. C. 1994, in IRAS Sky Survey Atlas Explanatory Supplement, by S. L. Wheelock, et al. (Pasadena, JPL), p. G-1

IRAS Catalogs and Atlases: Explanatory Supplement 1988, ed. C. A. Beichman, G. Neugebauer, H. J. Habing, P. E. Clegg, and T. J. Chester (Washington, DC, GPO)

IRAS Point Source Catalog, Version 2 1988, Joint IRAS Science Working Group (Washington, DC, GPO)

Jaynes, E. T. 1986, in Maximum Entropy and Bayesian Methods in Applied Statistics, ed. J. H. Justice (Cambridge, Cambridge University Press), p. 26

Lucy, L. B. 1974, AJ, 79, 745

Maccabe, B., McCurley, K. S., and Riesen, R. 1993, SUNMOS for the Intel Paragon, ftp://cs.sandia.gov/pub/sunmos/doc

Pierro, A. R. De 1991, in Mathematical Methods in Tomography, ed. G. T. Herman, A. K. Louis, and F. Natterer (Berlin, Springer), p. 167

Press, W. H., Teukolsky, S. A., Vetterling, W. T., and Flannery, B. P. 1992, Numerical Recipes in C, 2nd ed. (Cambridge, Cambridge University Press), p. 823

Rice, W. 1993, AJ, 105, 67

Richardson, W. H. 1972, JOSA, 62, 55

Scoville, N. Z., and Good, J. C. 1989, ApJ, 339, 149

Skilling, J. 1986, in Maximum Entropy and Bayesian Methods in Applied Statistics, ed. J. H. Justice (Cambridge, Cambridge University Press), p. 26

Sodroski, T. J., Dwek, E., Hauser, M. G., and Kerr, F. J. 1989, ApJ, 336,762

Soifer, B. T., Houck, J. R., and Neugebauer, G. 1987, ARAA, 25, 187

Snell, R. L., Heyer, M. H., and Schloerb, F. P. 1989, ApJ, 337, 739

Surace, J. A., Mazzarella, J. M., Soifer, B. T., and Wehrle, A. E. 1993, AJ, 105, 864

Terebey, S., and Fich, M. 1986, ApJ, 330, L73

Terebey, S., and Mazzarella, J., eds. 1994, Science with High Spatial Resolution Far-Infrared Data (Pasadena, JPL)

Weiland, J. L., Blitz, L., Dwek, E., Hauser, M. G., Magnani, L., and Richard, L. J. 1986, ApJ, 306, L101

Wheelock, S. L., et al. 1994, IRAS Sky Survey Atlas Explanatory Supplement (Pasadena, JPL)

Wood, D. O. S., Myers, P. C., and Daugherty, D. A. 1994, ApJS, 95, 457

Zervakis, M. E., and Venetsanopoulos, A. N. 1992, Multidimen. Syst. Signal Process., 3, 381 